\title{
Development of Stand Alone Application Tool for Processing and Quality Measurement of Weld Imperfection Image Captured by $\mu$-Focused Digital Radiography Using MAT- LAB-Based Graphical User Interface
}

\author{
PZ Nadilaa, YHP Manurung*a, SA Halim ${ }^{\text {b }}$ SK Abas ${ }^{\mathrm{a}}$, G Thama, E Harumanc, M Mokhtard and \\ Z Awaldine \\ ${ }^{a}$ Faculty of Mechanical Engineering, Universiti Teknologi MARA (UiTM), Selangor, Malaysia. \\ ${ }^{b}$ Faculty of Computer and Mathematical Sciences, Universiti Teknologi MARA (UiTM), Malaysia. \\ ${ }^{c}$ Faculty of Industrial Engineering, Bakrie University, Jakarta, Indonesia. \\ ${ }^{d}$ School of Mechanical Engineering, Engineering Campus, Universiti Sains Malaysia (USM), Malaysia. \\ eZL Technologies Sdn. Bhd, No. 32, Jln Nilam 1/1, Taman Teknologi Tinggi Subang, 40000 Shah Alam, Malaysia.
}

Received 10 April 2011; accepted 15 February 2012

\begin{abstract}
Digital radiography incresingly is being applied in the fabrication industry. Compared to film- based radiography, digitally radiographed images can be acquired with less time and fewer exposures. However, noises can simply occur on the digital image resulting in a low-quality result. Due to this and the system's complexity, parameters' sensitivity, and environmental effects, the results can be difficult to interpret, even for a radiographer. Therefore, the need of an application tool to improve and evaluate the image is becoming urgent. In this research, a user-friendly tool for image processing and image quality measurement was developed. The resulting tool contains important components needed by radiograph inspectors in analyzing defects and recording the results. This tool was written by using image processing and the graphical user interface development environment and compiler (GUIDE) toolbox available in Matrix Laboratory (MATLAB) R2008a. In image processing methods, contrast adjustment, and noise removal, edge detection was applied. In image quality measurement methods, mean square error (MSE), peak signal-to-noise ratio (PSNR), modulation transfer function (MTF), normalized signal-to-noise ratio $\left(\mathrm{SNR}_{\text {norm }}\right)$, sensitivity and unsharpness were used to measure the image quality. The graphical user interface (GUI) wass then compiled to build a Windows, stand-alone application that enables this tool to be executed independently without the installation of MATLAB.
\end{abstract}

Keywords: Digital radiography, Modulation transfer function, Peak signal-to-noise ratio, Mean squared error, Matrix laboratory

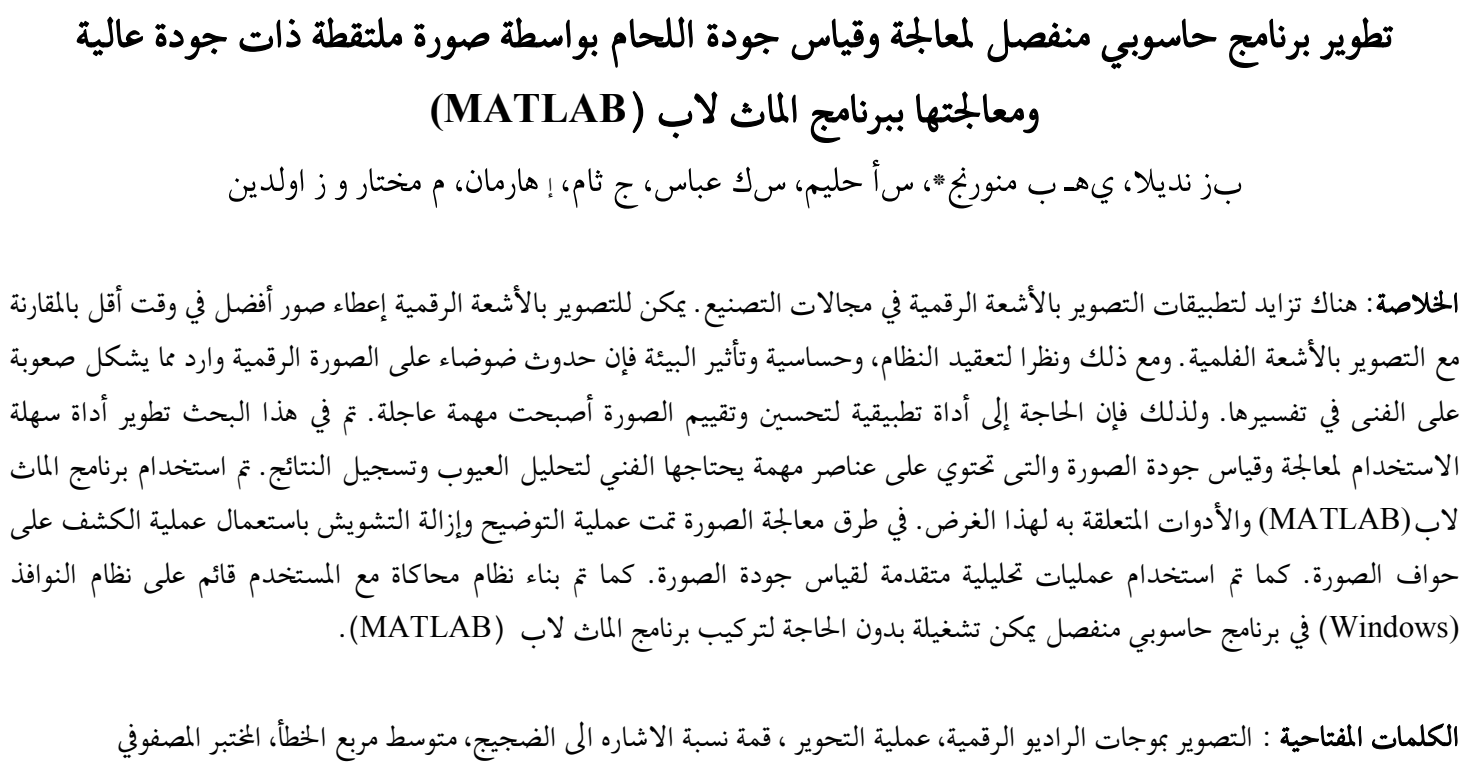




\section{Introduction}

Non-destructive testing (NDT) is an applied technology for inspecting materials. Other common applications are to assist in product development, screen or sort incoming materials, monitor, improve or control manufacturing processes, and inspect for in-service damage. Radiography testing is one the most commonly used NDT techniques in welding industries (Pardikar 2008). Since the discovery of X-rays in 1895 , film has been the primary medium for capturing, displaying, and storing radiographic images. The processing of X-ray film includes development, fixing, washing and drying to obtain an image that shows the defect. An inspector is then required in the interpretation process for the acceptance based on standard or industry requirements.

Nowadays, digital radiography is progressively replacing conventional, film-based radiographic techniques because it provides higher image quality, more effective use of radiation, and more efficient work practice thanks to the ability in affords to by pass chemical processing. Digital radiography also allows for digital file transfers and easy enhancement of images. Furthermore, less radiation is required to produce an image of similar quality to those produced by conventional radiography. Digital radiography is a form of X-ray imaging, where digital X-ray sensors such as charge-coupled devices (CCD) and flat-panel detectors (FPD) are used instead of traditional radiographic film. A comparison between film-based and digital radiography image properties is summarized in Table 1 (Edwin and Williamson 2002).

Recently, numerous software programs such as Quick MTF, I See (Noorhazleena 2010), ImageJ, and Imatest have emerged and serve to improve and evaluate image quality. However, there still exists a deficit in the market a comprehensive analyzing and reporting tool that is particularly tailored to the needs of the modern welding industry. In this investigation, an application tool for image processing and image quality is designed for analyzing welding imperfections captured by digital radiography. The following article is comprised of three main sections (1) introduction and theoretical background (2) image processing method and (3) image quality measurement methods. In the first part, the theoretical background of the tool, is introduced. In the second part, of image processing methods for noise removal, intensity adjustment, and edge detection are disdussed. The third part image quality measurement, is used to analyze the image based on modulation transfer function (MTF), image sensitivity using image quality indicators (IQI), single wire (10 FE EN), image unsharpness using IQI duplex wire (EN 462-5), and normalized signal noise ratio $\left(\mathrm{SNR}_{\text {norm }}\right)$ using a step wedge.

\section{Basic Principle and System Overview of $\mu$-Focused Digital Radiography}

In this investigation, images were acquired using a NDT analyzer model: m-225D (GE Phoenix X-ray), Systems, Wunsdorf, Germany) with a digital image chain for enhanced contrast and superior resolution. This $\mu$-focused digital radiography has 9" triple-, 6" dual- and 6" single-field image intensifiers. Radiographs were acquired using a $1000 \times 1000$ pixelCCD camera with a 12-bit image format. Figure 1 shows an overview of the image intensifier system. The incoming X-rays are converted into a visible light. A photo cathode converts the visible light into electrons which are then accelerated and focused onto a fluorescent screen. On the screen, a bright, small and visible X-ray image appears and is received by the CCD camera. An X-ray tube is used to control the radiation for higher detectability of small details of weld defects. A smaller focus size indicates smaller geometrical unsharpness to produce a sharper image (Nadila et al. 2010). Figure 2 shows $\mu$-focused digital radiography equipment, its system overview and a sample of a radiographed image. Equation (1) shows the relationship between focus spot size (f), focus-todetector distance (FDD), focus-to-object distance (FOD), and geometrical unsharpness (U).

$$
U=f \times \frac{F D D-F O D}{F O D}=f \times \frac{b-a}{a}
$$

\section{MATLAB-Based Graphical User Inter- face Development}

In graphical user interface (GUI) development, the image processing method and the corresponding tool for the measurement of image quality were designed and developed by using three main tool boxes incorporated in the MATLAB Graphical User Interface Development Environment and Compiler (GUIDE). The image processing toolbox has a variety of methods which can be used to enhance the image. The generated codes were integrated within the GUI to develop a complete function for the interface of the application tool. The developed GUI was then compiled using the MATLAB compiler. A standalone application is an executable program that includes a selection of Windows standalone applications adding the $\mathrm{m}$-file to main functions, building the $\mathrm{m}$-file, and then packaging the compiled file. Figure 3 shows a model development process that explains the development of GUI up to the execution process.

\subsection{Introduction and Theoretical Background}

This section presents essential information regard- 
Development of Stand Alone Application Tool for Processing and Quality Measurement of Weld Imperfection Image Captured by $\mu$-Focused Digital Radiography Using MATLAB-Based Graphical User Interface

Table 1. Comparison between conventional and digital X-ray

\section{Conventional X-Ray}

Contrast is the difference in densities between various areas on a radiograph; high contrast images have few shades of gray between black and white while low contrast will demonstrate more shades of gray

Resolution is the ability to distinguish between small objects that are close together; measured in line pairs per millimeter

Density is the overall degree of darkening of an exposed film

Sharpness is the ability of a radiograph to define an edge or display density boundaries

Require higher radiation exposure.

Focal Spot Size of conventional units is greater than 200 microns

Image acquired from the film directly without any special image processing techniques that can enhance overall image.

\section{Digital X-Ray}

Contrast resolution is the ability to differentiate small differences in density as displayed on an image

Spatial frequency is to measure of resolution expressed in line pairs per millimeter. MTF is to measure image fidelity as a function of spatial frequency (How close is the image to the actual object?).

Brightness is equivalent to density or overall degree of image darkening.

Signal to noise ratio-ratio (SNR) between the fraction of the output signal (voltage or current or charge) that is directly related to the information (signal) and the fraction of output that does not contain information (noise).

Less radiation exposure can be used to produce an image of similar con trast to conventional radiography.

Focal Spot Size of $\mu$-focused digital radiography is smaller than 50 microns which is enable to achieve the best resolution.

Immediate image preview and availability, a wider dynamic range which makes it more forgiving for overand under-exposure as well as the ability to apply special image processing techniques that enhance overall image.

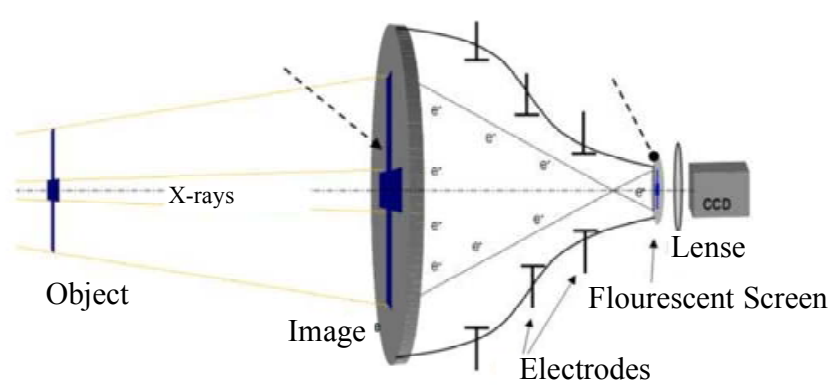

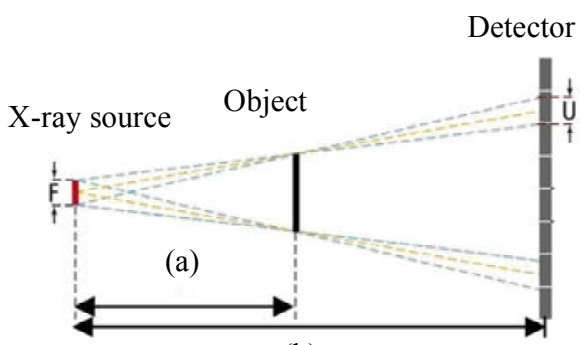

(b)

Figure 1. System overview of image intensifier (left) and unsharpness (U) on the detector (right)
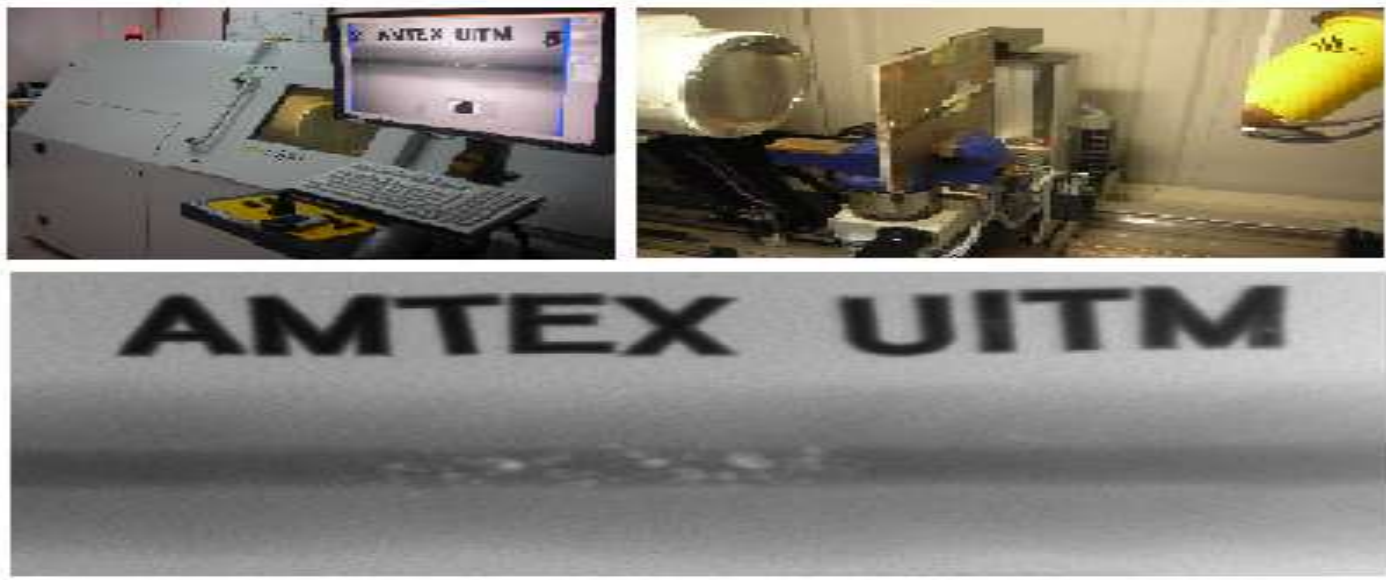

Figure 2. $\mu$-focussed digital radiograph (top, left), system overview (top, right) and radiographed image using flawed specimen (below) using $180 \mathrm{~V}, 240 \mathrm{~A}, \mathrm{FDD}=870 \mathrm{~mm}$ and FOD $=270 \mathrm{~mm}$ 


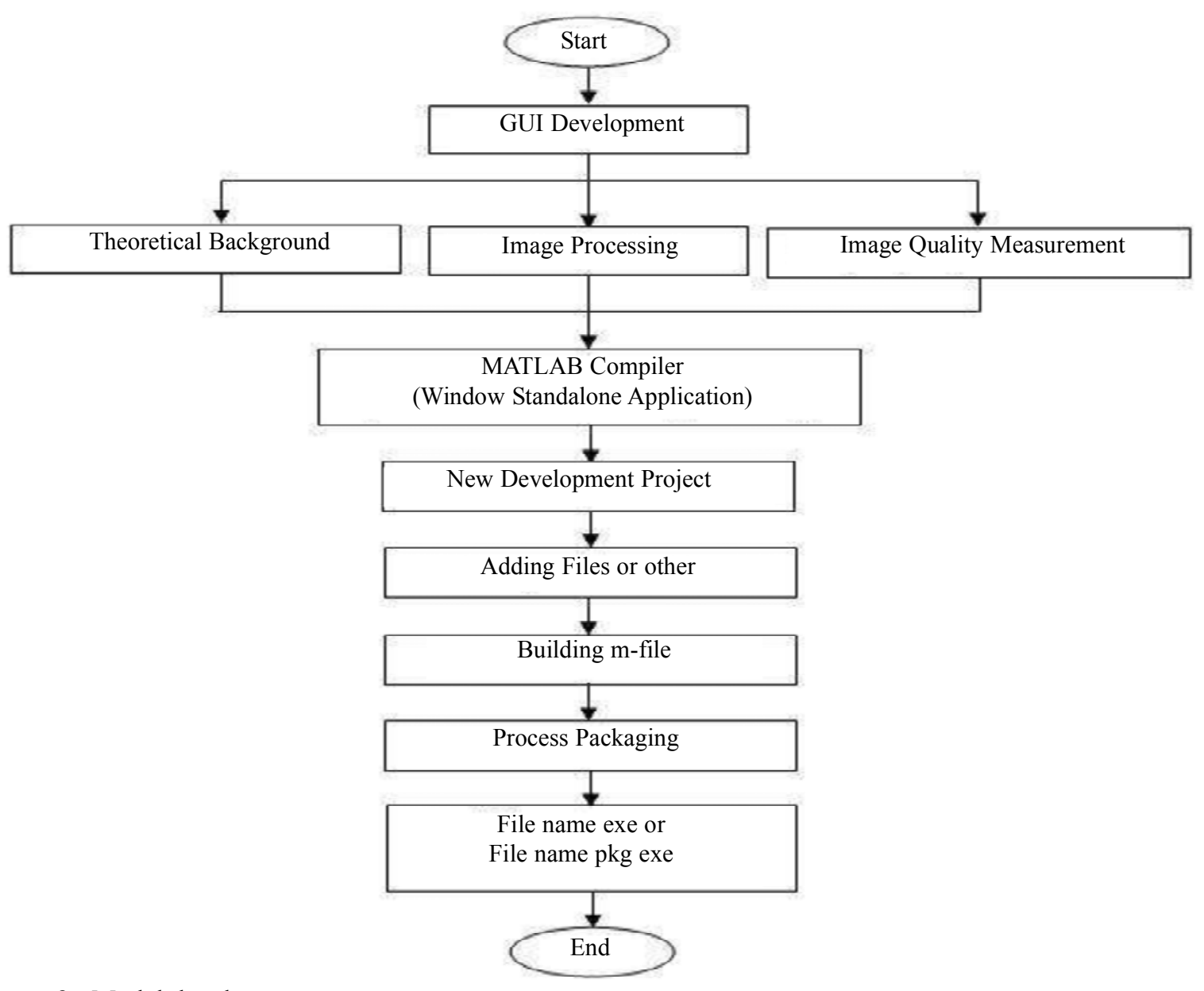

Figure 3. Model development process

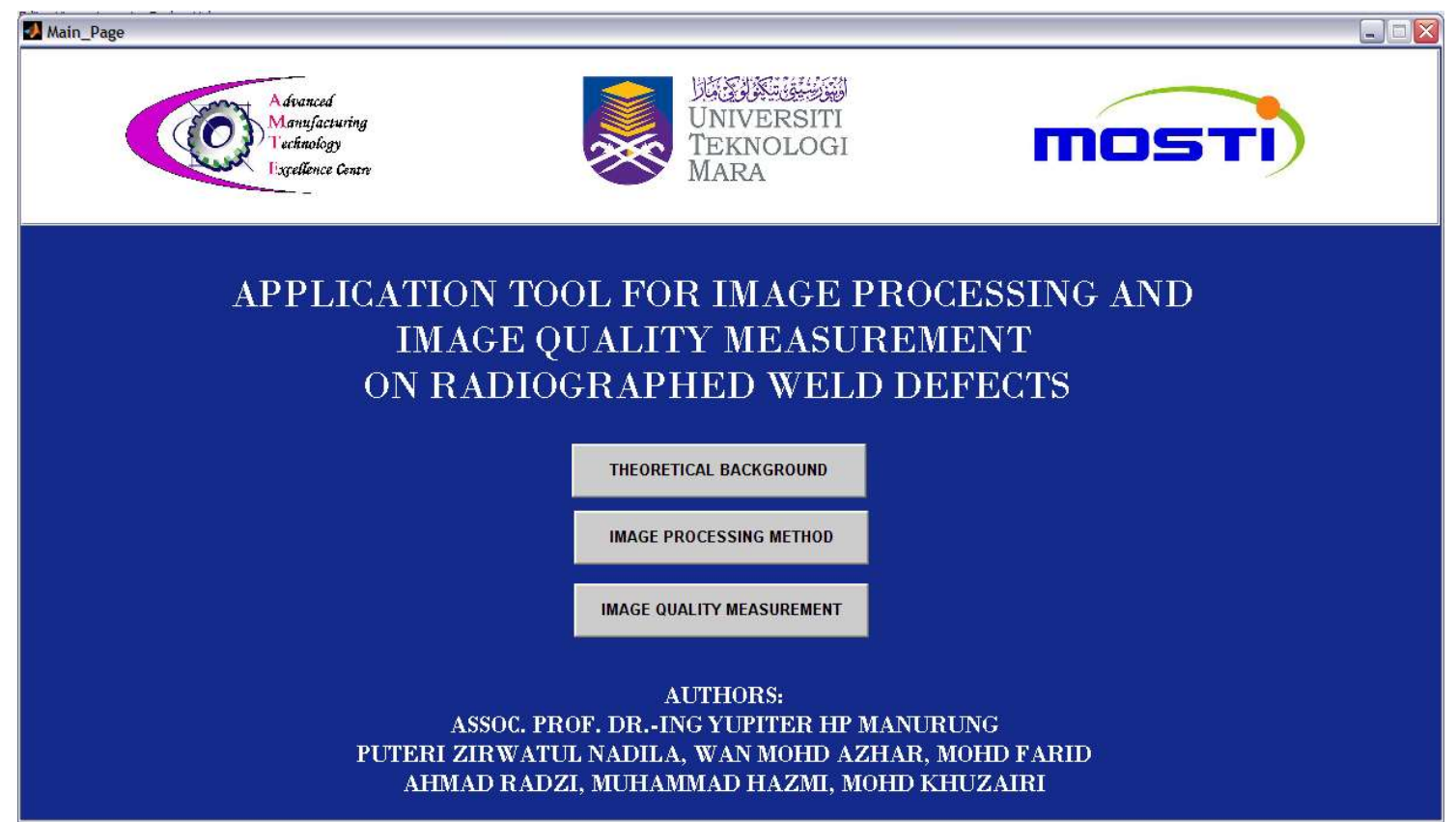

Figure 4. Main page of the GUI-based application tool

ing the basic principles of the NDT methods and filmbased and digital radiography, a system overview of $\mu$ focused technology, various methods of image pro- cessing and image quality measurement, and collections of experimental results using flawed specimens. In this section, a user or interpreter could easily gain 

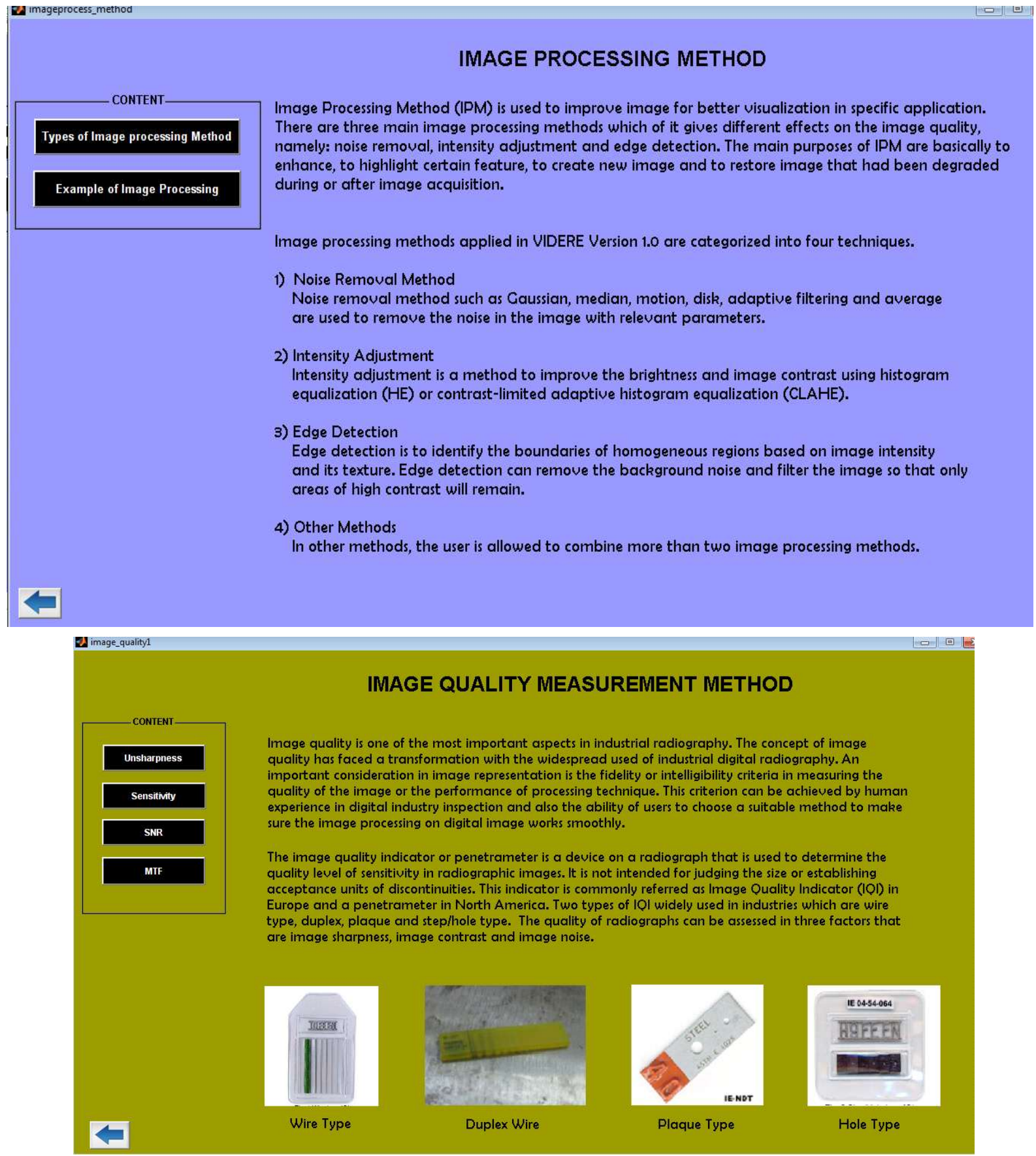

Figure 5. Example of content in theoretical background

comprehensive information prior to GUI operation. Figure 4 shows the main page of image processing and image quality measurement tools that provide three main parts, namely theoretical background, an image processing method, and image quality measurement techniques. In addition, Figure 5 shows the contents of each part.

\subsection{Image Processing Method (IPM)}

The image processing method (IPM) is divided into four parts which are noise removal, intensity adjustment, edge detection, and other methods. The main purpose of these methods is to bring out the details of an image. A low-contrast image can be adjusted by modifying the pixel intensity of the input image (Xie et al. 2009). The methods that were selected for this process are based on the MATLAB image processing toolbox which contains Gaussian, adaptive filtering, medians, etc. It is important to choose a suitable image processing method due to the requirements of welding image enhancement is to improve image quality and vision effects which act as the foundation of a welding defects analysis (Han et al. 2009). Figure 6 shows the components in IPM, including (1) an image before and 


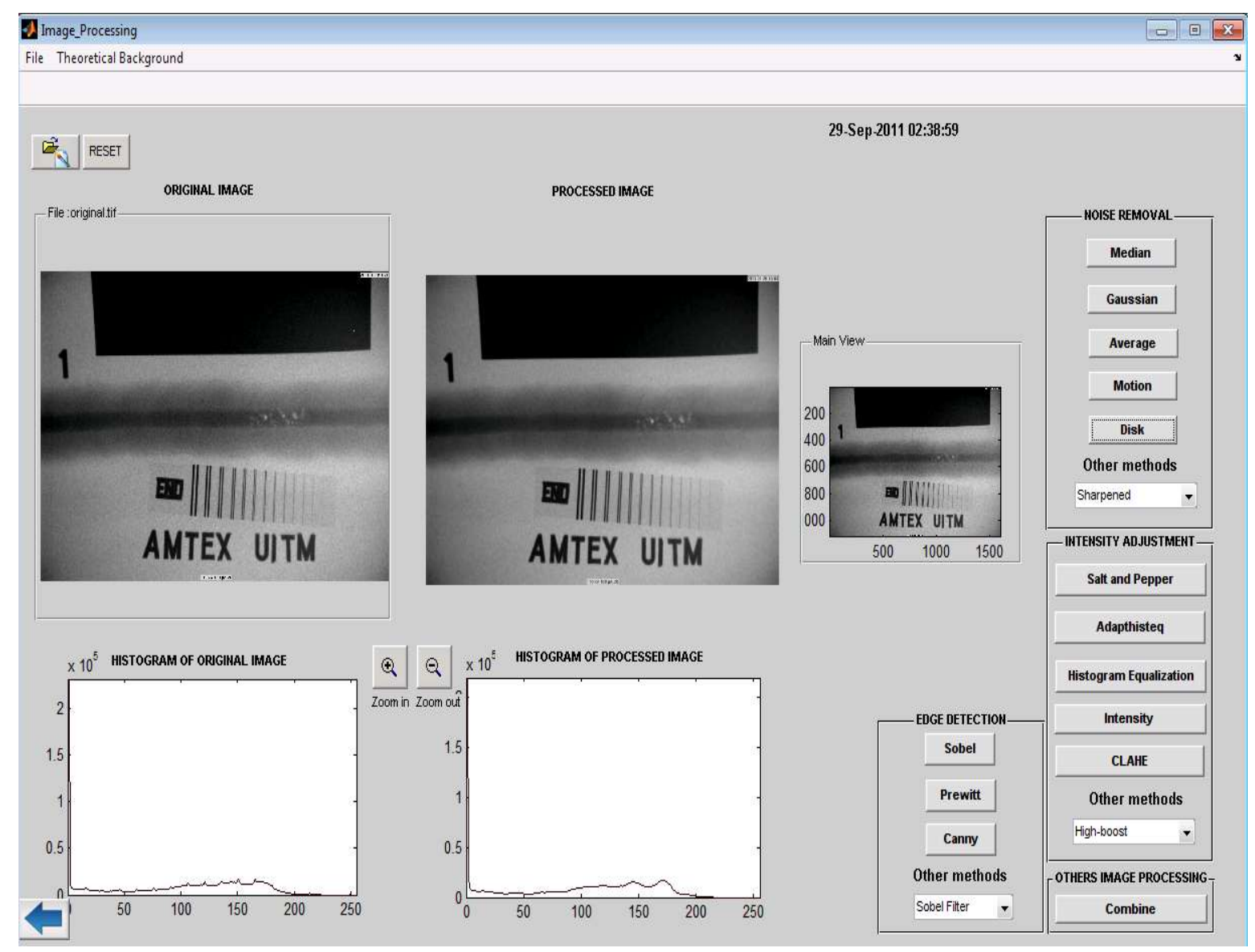

Figure 6. Image processing method (IPM) interface. (1) Image before and after processed with its histogram, (2) View window and (3) Selection menu of image processing method

after being processed with its histogram (2) a view window, and (3) the selection menu of the IPM.

The principle of enhancement techniques is to process an image so that the result is more suitable than the original image for the specific application (Rafael and Richard 1992). The IPM consists of two main parts, which are noise removal and intensity adjustment by histogram equalization.

\subsubsection{Noise Removal Method}

Noise removal provides a smoothing effect on the image. A median filter is an alternative approach to reduce the noise that preserves better edge by replacing the grey level of each pixel with the median of the grey levels in the neighborhood of that pixel. The MATLAB functions of imnoise and medfilt removed the noise with less edge blurring. Imnoise represents as randomly occurring white and black pixel, which is then restored by medfilt2, to reduce noise and preserve the edge. The median filter is represented using Eqn. (2), where $N(x, y)$ is the immediate neighbors of pixel $(x, y)$ :

$$
g^{\prime}(x, y)=\operatorname{median}\{g(x, y) \text { is in } N(x, y)\}
$$

The Gaussian filter can be shaped by the user in terms of size and standard deviation. A Gaussian low pass filter can remove noise sufficiently by using Eqn. (3). where $u$ is the standard deviation of the Gaussian filter.

$$
G(x, y)=e^{\frac{-\frac{1}{2} u^{2}}{u^{2}}}
$$

The average filter replaced the value of the center pixel by averaging the value of the neighborhood pixels. The image after neighborhood smoothing is illustrated by Eqn. (4), where $M$ is the total points of the neighborhood pixel and $S$ is a neighborhood of point $(x, y)$.

$$
g^{\prime}(x, y)=\frac{1}{M} \sum_{x, y \in S} f(x, y)+\frac{1}{M} \sum_{x, y \in S} n(x, y)
$$

An adaptive filter is a class of filters that change their characteristics according to the values of the gray scales under the mask by using the local statistical properties. A wiener filter (wiener 2) is one of the 
adaptive filters represented using the Eqn. (5), where $S_{x x}\left(f_{1}, f_{2}\right), S_{\eta \eta}\left(f_{1}, f_{2}\right)$ and indicate respectively the power spectra of the original image and the additive noise, and $H\left(f_{1}, f_{2}\right)$ is the blurring filter.

$$
W\left(f_{1}, f_{2}\right)=\frac{H^{*}\left(f_{1}, f_{2}\right) S_{x x}\left(f_{1}, f_{2}\right)}{\left|H\left(f_{1}, f_{2}\right)\right|^{2} S_{x x}\left(f_{1}, f_{2}\right)+S_{\eta \eta}\left(f_{1}, f_{2}\right)^{\prime}}
$$

A disk filter or circular averaging filter (pillbox) is also a method used in an image processing tool and provides a blurring effect. It is defined by Eqn. (6), where, $R$ is a defocused radius. Increasing the radius of the filter can give more of a blurring effect on the image. The value of the radius chosen for this method was 3 .

$$
h(x, y)=\left\{\begin{array}{cc}
0 ; & \sqrt{x^{2} \pm y^{2}}>R \\
1 /\left(\pi R^{2}\right) & \sqrt{x^{2} \pm y^{2}} \leq R
\end{array}\right.
$$

\subsubsection{Contrast Enhancement Method}

An important method that can be used to alter the image is histogram equalization (HE). HE is widely used for contrast enhancement to improve image brightness and to provide an effect on the dynamic range stretching (Yeong-Taekgi 1997). The HE method usually increases the global contrast of images, especially when the usable data of the image is represented by close contrast values. Through the adjustment, the intensities can be better distributed on the histogram. This allows areas of lower local contrast to gain higher contrast. HE accomplishes this by effectively spreading out the most frequent intensity values. Thus, it can increase the contrast quality with the result that the weld discontinuities on the film can to be clearly seen. The formula for histogram equalization is given in Eqn. (7), where $r_{k}$ is the input intensity, $S_{k}$ is the processed intensity, $k$ is the intensityand range (0.0-1.0), $n_{j}$ is the frequency of intensity, and $j$ and $n$ is the sum of all frequencies.

$$
S_{k}=T\left(r_{k}\right)=\sum_{j=1}^{k} p_{r}\left(r_{j}\right)=\sum_{j=1}^{k} \frac{n_{j}}{n}
$$

As a result, the dynamic range of the histogram is stretched evenly flat where the gray levels have uniform probability density. Figure 7 shows the interface of the contrast enhancement results after using HE.

\subsection{Image Quality Measurement Method (IQM)}

The quality of the enhanced image is further analyzed in terms of noise, sensitivity, unsharpness, mod- ulation transfer function (MTF), and the ratio of signal to noise. The noise is measured based on the mean square error (MSE) and peak single-to-noise ratio (PSNR). Besides that, the image quality indicator (IQI) has been used to evaluate the sensitivity and unsharpness of the image. The MTF is measured on a region of interest (ROI) on a tungsten plate, which produces a clear, distinct edge. The measurement of signal-to-noise ratio (SNR) is carried out on a step wedge with five (5) different thicknesses. The quality measurements that are used to measure the image quality are summarized as follow:

\section{(a) MSE and PSNR}

Besides visual evaluation by a human interpreter, the performance of an enhanced image can be evaluated quantitatively using MSE and PSNR. The MSE represents the cumulative squared error between the processed and the original image, whereas PSNR represents a measure of the peak error and is measured in decibel (dB) units. Eqns. (8) and (9) show the equation for MSE and PSNR, where $M$ and $N$ are the height and width of the image respectively. $I(x, y)$ is the original image and $I^{\prime}(x, y)$ is the processed image.

$$
\begin{aligned}
& M S E=\sum_{y=1}^{M} \sum_{x=1}^{N}\left[I(x, y)-I^{\prime}(x, y)\right]^{2} \\
& P S N R=10 \log _{10}\left(\frac{R^{2}}{M S E}\right)
\end{aligned}
$$

PSNR is the ratio of the peak signal power to the average noise power. It represents the ratio between a maximum of the signal $\left(R^{2}\right)$ and the MSE ([I $(x, y)$ and $\left.\left.I^{\prime}(x, y)\right]\right) . \quad R^{2}$ is the maximum peak-to-peak swing of the signal, whereby $R$ is 255 for 8 -bit images. The processed image is considered better quality when the MSE is low and the PSNR is a high in value.

\section{(b) Measurement of Sensitivity and Image Unsharp- ness using IQI}

IQI is used to control the quality of a radiograph that can improve visualization on the film. Two common types of IQI for assessing radiographic quality are IQI single wire (10 FE EN) and IQI duple wire (EN462-5). For IQI single wire, the last visible wire on a radiographic image is considered as the contrast sensitivity of the wire which can be calculated using Eqn. (10).

$\%$ Sensitivity $=\frac{\text { Diameter of thinnest discernible wire }}{\text { Material thickness }} \times 100 \%$ 


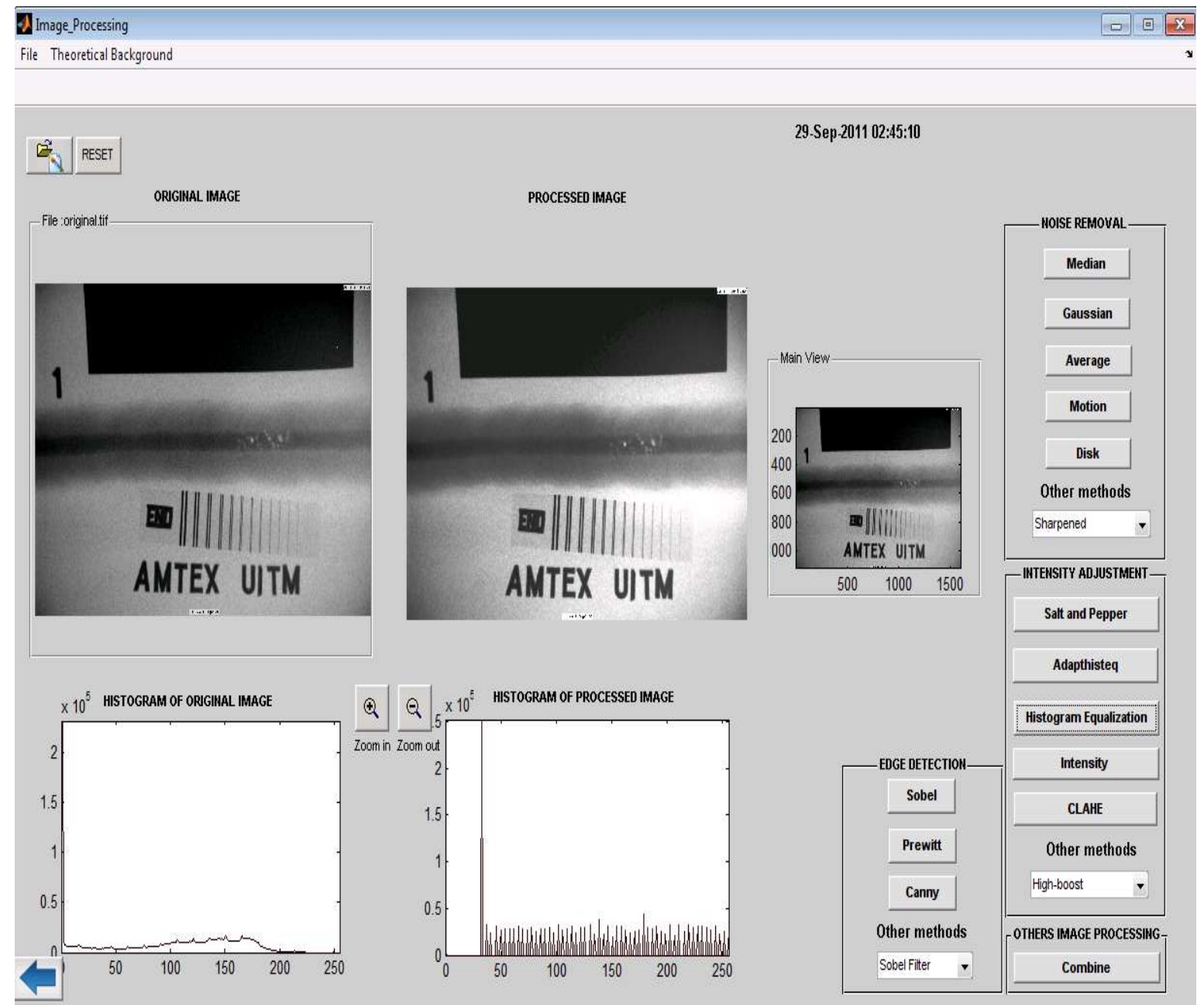

Figure 7. Contract enhancement result (Histogram after HE process)

Image unsharpness is one of the image quality parameters that is important in evaluating the system properties and qualities. It can be achieved by taking the geometric unsharpness divided by the magnification. Detector unsharpness is determined by the basic spatial resolution $\left(\mathrm{SR}_{\mathrm{b}}\right)$ that corresponds to the effective pixel size. Standard committees, recommended use of the duplex wire method due to its simplicity (EN 462-5, ISO 19232-5 and ASTM E 2002) (Uwe et al. 2007). By referring to the IQI duplex wire table Table 2, the total unsharpness and SRb can be determined using Eqn. (11).

$$
S R_{b}=\frac{\text { Total Unsharpness }(T U)}{2}
$$

\section{(c) Measurement of MTF and Spatial Frequency}

The MTF of an imaging system measures the sine waves that describe the transmission of $\mathrm{X}$-rays through an object. The transmitted X-ray beam is modulated by the object being inspected, and the imaging system is responsible for translating the signal into a visible image. The MTF is represented by an curve with the spatial frequency response and measured in line pairs per millimeter $(\mathrm{lp} / \mathrm{mm})$ or cycles per millimeter (c/mm) (Handee and Russell 2002). In this investigation, a ROI on a tungsten plate that provides a clear distinct edge is determined to calculate the MTF. The MTF of an imaging system can be determined by the edge spread function (ESF) and line spread function (LSF). The ESF is differentiated to obtain the LSF (Samei and Flynn 2003). ESF uses a sharp edge rather than simply a line. It is measured when various influences to the imaging system are dependent on the area exposed, such as when the spatial properties of scattered X-radiation are measured, or for systems that have a large amount of optical light scatterings, such as fluoroscopy. The ESF formula is given in Eqn. (12), where $x$ is the direction perpendicular to the edge, $\mathrm{x}_{0}$ is the location of the edge, $-B$ and $B$ are the left and right boundaries of the image and $A$ is constant.

$$
\begin{array}{r}
\operatorname{ESF}(\mathrm{x})=0 \quad \mathrm{x}_{0}>\mathrm{x} \geq-\mathrm{B} \\
\mathrm{A} \quad \mathrm{B} \geq \mathrm{x} \geq \mathrm{x}_{0}
\end{array}
$$


LSF can be considered as a line of continuous holes placed very close together. Mathematically, the line spread function is the first derivative of the ESF. LSF is given by Eqn. (13).

$$
\operatorname{LSF}(x)=\frac{d[E S F(x)]}{d x}
$$

High spatial frequency means more line pairs (black and white stripes) can be observed in one millimeter. The Fourier transforms (FT) method changes the pixel form to spatial frequency in terms of a line pair per millimeter $(\mathrm{lp} / \mathrm{mm})$. In most cases, FT is used to convert images from the spatial domain into the frequency domain and vice-versa. The FT is also an important image processing tool that is used to decompose an image into its sine and cosine components. The output of the transformation represents the image in the Fourier or frequency domain, while the input image is the spatial domain equivalent. In the Fourier domain image, each point represents a particular frequency contained in the spatial domain image. The MTF equation is given in Eqn. (14).

$$
\operatorname{MTF}(f x)=\frac{1}{\int_{=\infty}^{+\infty} L S F(x) d x}\left|\int_{=\infty}^{+\infty} L S F(x) \times e^{-2 \pi j(x f x)} d x\right|
$$

(d) Measurement of Normalized Signal Noise Ratio $\left(S N R_{\text {norm }}\right)$

SNR is a technical term used in digital radiography to quantify the amount of corruption signal caused by noise. The SNR imposes the fundamental limitation of object perceptibility in a radiograph because image contrast can be manipulated during the display of digitally acquired radiographic images. The SNR is given by the ratio of the light signal to the sum of the noise signals and measured in decibels $(\mathrm{dB})$ units. The SNR equation is given in Eqn. (15), where $M_{s w x, s w y}(x, y)$ is a

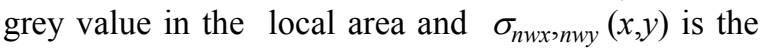
square root of window variance.

$$
S N R=\frac{\text { signal }_{\text {est }}}{\text { noise }_{\text {est }}}=\frac{M_{\text {swx,swy }}(x, y)}{\sigma_{n w x, n w y}(x, y)}
$$

Theoretically, SNR increases with the square root of the detector's pixel area under the same radiation quality and exposure time (Ewert et al. 2007). The standard pixel area should be normalized using $\mathrm{SNR}_{\text {norm }}$ to allow a comparison of different detectors. The proposed equivalent square pixel area is $88.6 \times 88.6 \mu \mathrm{m}^{2}$ and the detector's basic spatial resolution, $\left(\mathrm{SR}_{\mathrm{b}}\right)$ is calculated by using Eqn. (11). The $\mathrm{SNR}_{\text {norm }}$ can be obtained using Eqn. (16).

$$
S N R_{\text {norm }}=S N R_{\text {measured }} \times \frac{88.6 \mu m}{S R_{b}}
$$

Experimentally, the $\mathrm{SNR}_{\text {norm }}$ is measured using the step wedge method (Pardikar 2008). Important parameters such as exposure time, current and voltage are used to acquire the image. Based on the theoretical and experimental facts, $\mathrm{SNR}_{\text {norm }}$ is inversed proportional to the thickness. The SNR is reduced as the thickness is increased. Figure 8 shows the layout of the IQM method and shows the MTF results.

\section{Execution, Operation and Results Using the Application Tool}

In this research, a flawed specimen from Sonaspection, (No. U-C-15) was captured and the radiographed image was enhanced using various methods. Prior to the calculation of ESF, LSF and MTF, the isotropic pixel spacing based on the detector specifications of the CCD camera and pixel subdivision are determined within a the range of 0.03 to 0.15 (Samei et al. 1998). Figure 9 shows the flow chart for the process involved in image processing and the IQM tool.

In this study, a flawed specimen with a thickness of $12 \mathrm{~mm}$ was made of carbon steel. The radiographed image of the specimen was captured using the parameters listed in Table 3. The original image was then processed using five different noise removal methods. The results are shown in Table 4.

Theoretically, a high PSNR and low MSE indicate a better result. This is due to the fact that the image has less signal loss after the enhancement with the image processing method. The result of the spatial frequency in $\mathrm{lp} / \mathrm{mm}$ (MTF) is obtained by defining a clear distinct edge on the image such as using a tungsten plate. Figure 10 shows the results of noise measurement of MSE and PSNR and spatial frequency in $1 \mathrm{p} / \mathrm{mm}$ with normalized SNR. Figure 11 shows the usage of a step wedge on different thicknesses of materials for $\mathrm{SNR}_{\text {norm }}$ calculation. Table 5 shows the results of the SNR and $\mathrm{SNR}_{\text {norm }}$ for four radiographed images with different thicknesses. The ninth wire in Figure 12 can be clearly seen. By referring to the IQI duplex wire table, the total unsharpness is $0.40 \mathrm{~mm}$.

Based on the results in Table 4, the original and enhanced images of a radiographic weld image was improved in terms of three parameters MSE, PSNR and MTF. Five methods of the noise removal show the smoothing effect on the radiographed image and the results show higher MTF values compared to the original image. Low values of MSE and high levels of PSNR were obtained indicating a quality improvement compared to the original image. 
PZ Nadila, YHP Manurung, SA Halim, SK Abas, G Tham, E Haruman, M Mokhtar and Z Awaldin
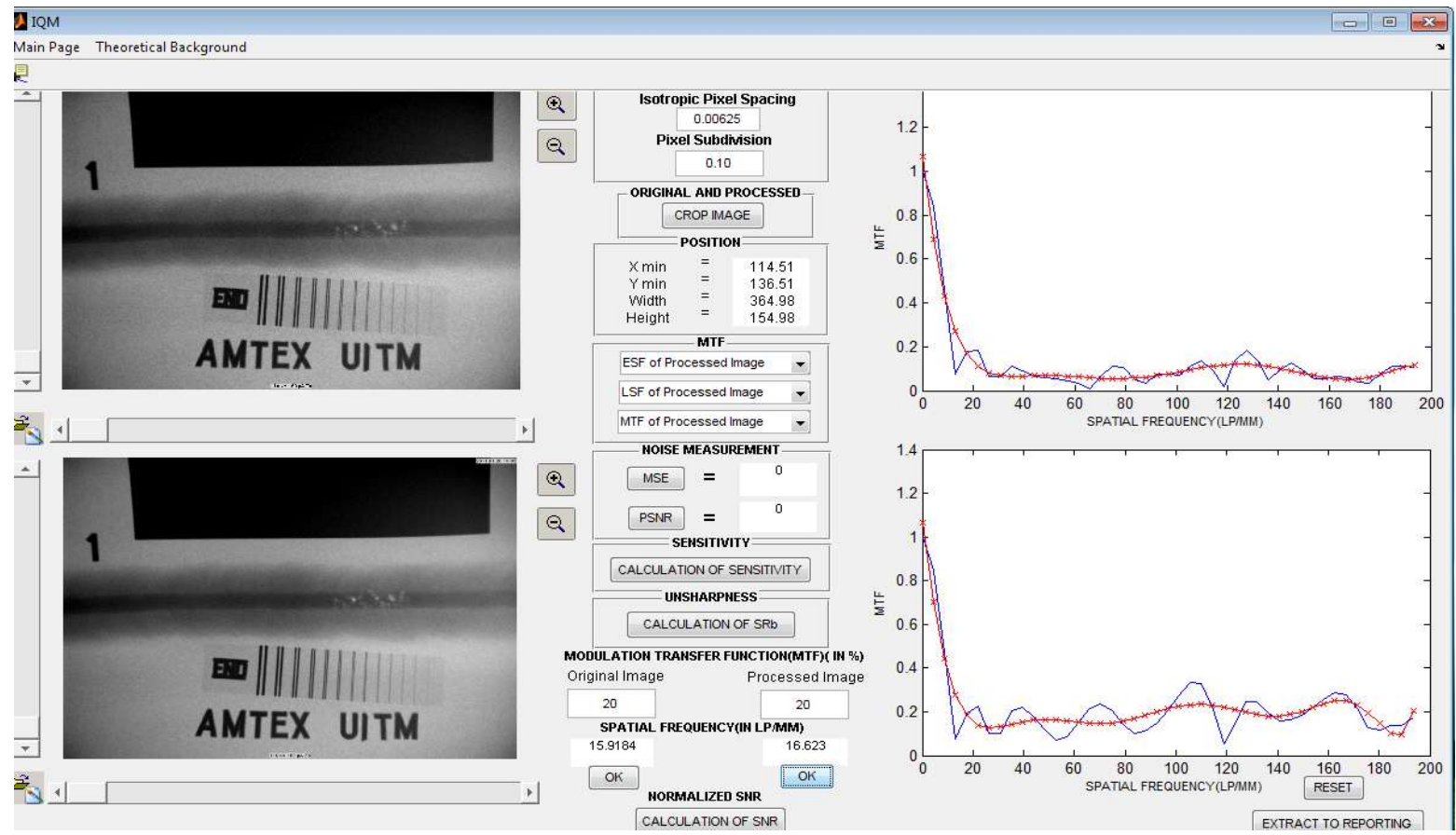

Figure 8. Results from image quality measurement: radiograph image before and after processed with RO1 (1) MTF plot (2)

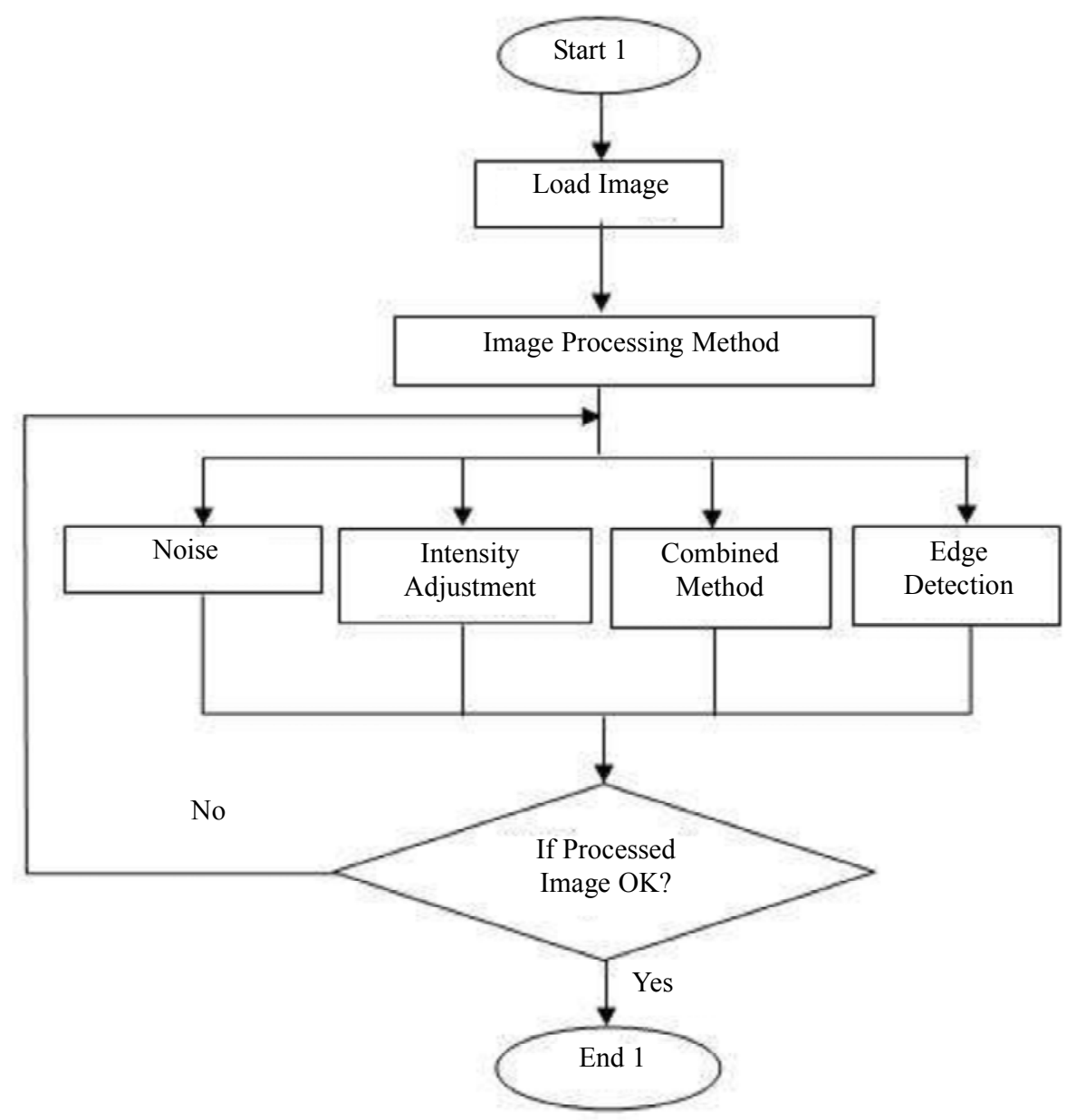




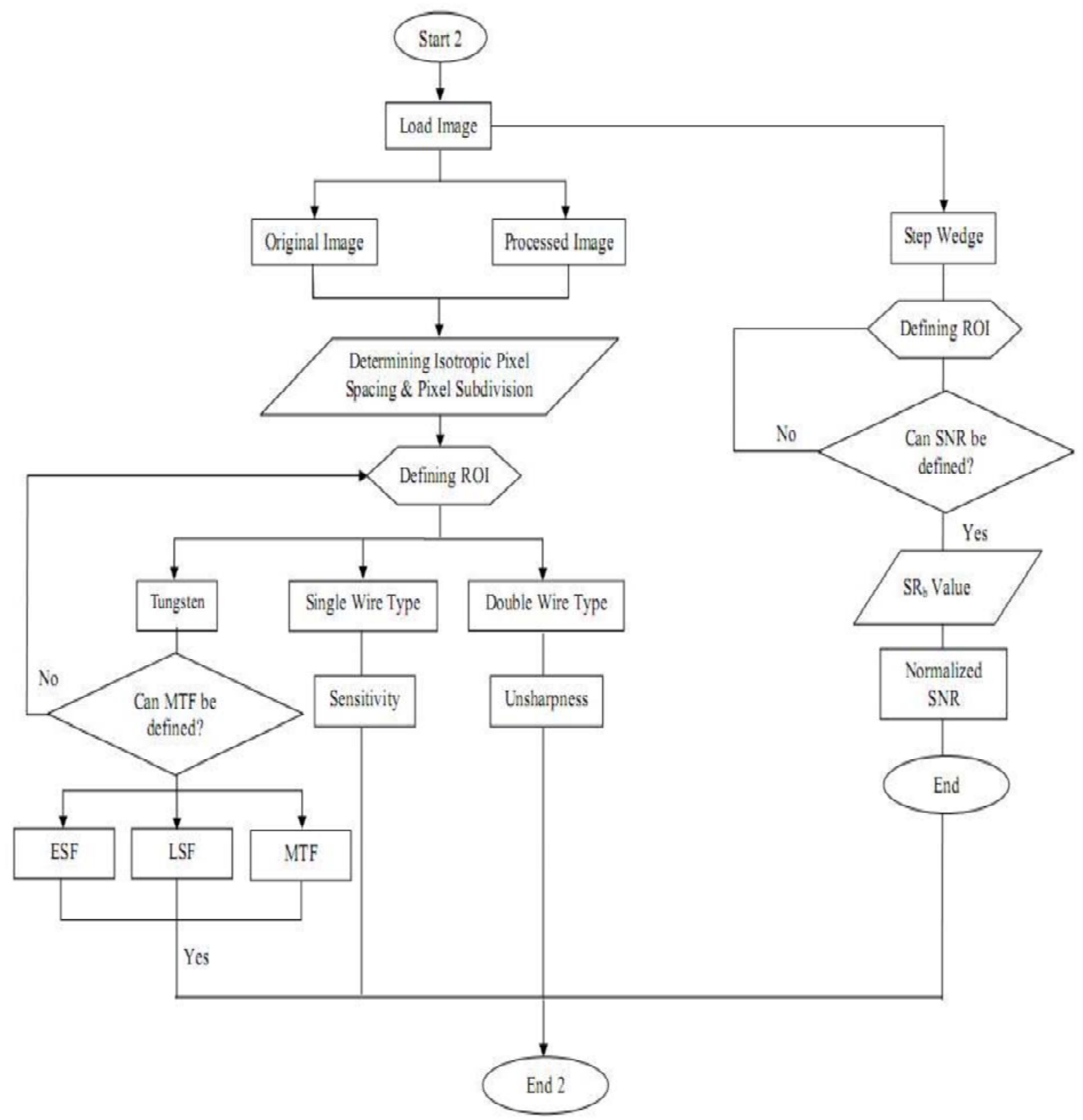

Figure 9. Operation of image processing (top) and image quality measurement (bottom)

\section{Conclusion and Further Recommenda- tion}

In this study, an application tool for image enhancement and image quality measurement was developed using MATLAB GUI. This tool allows the user to determine the parameter value, select methods for image enhancement, and display the respective results. The results show some improvement on the radiographed image based on MSE and PSNR. The processed images show increasing spatial frequency at $20 \%$ MTF by using all methods of noise removal as shown in Table 4. MATLAB-based GUI provides good performance in developing this application tool in the matter of time and cost effectiveness. Due to the fact that the record of welding results is an important matter in the industry, a comprehensive application tool that includes a reporting tool should be further developed. It is predicted that the future of radiography will be digital in the welding fabrication industry, therefore, it would behoove the interpreter or operator should be familiar with technical principles and image quality criteria. 
PZ Nadila, YHP Manurung, SA Halim, SK Abas, G Tham, E Haruman, M Mokhtar and Z Awaldin

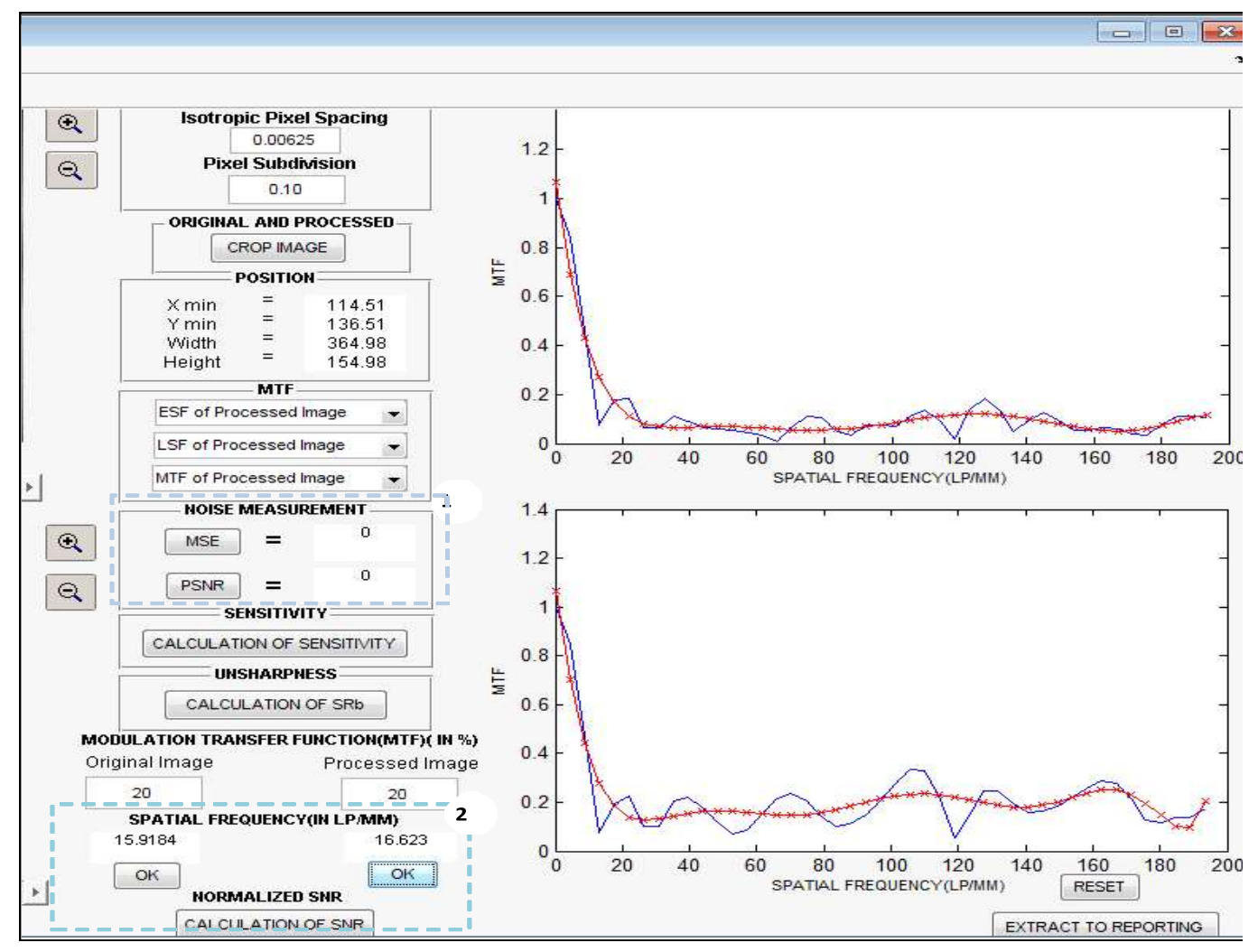

Figure 10. Results of noise measurement (1) and spatial frequency (2)
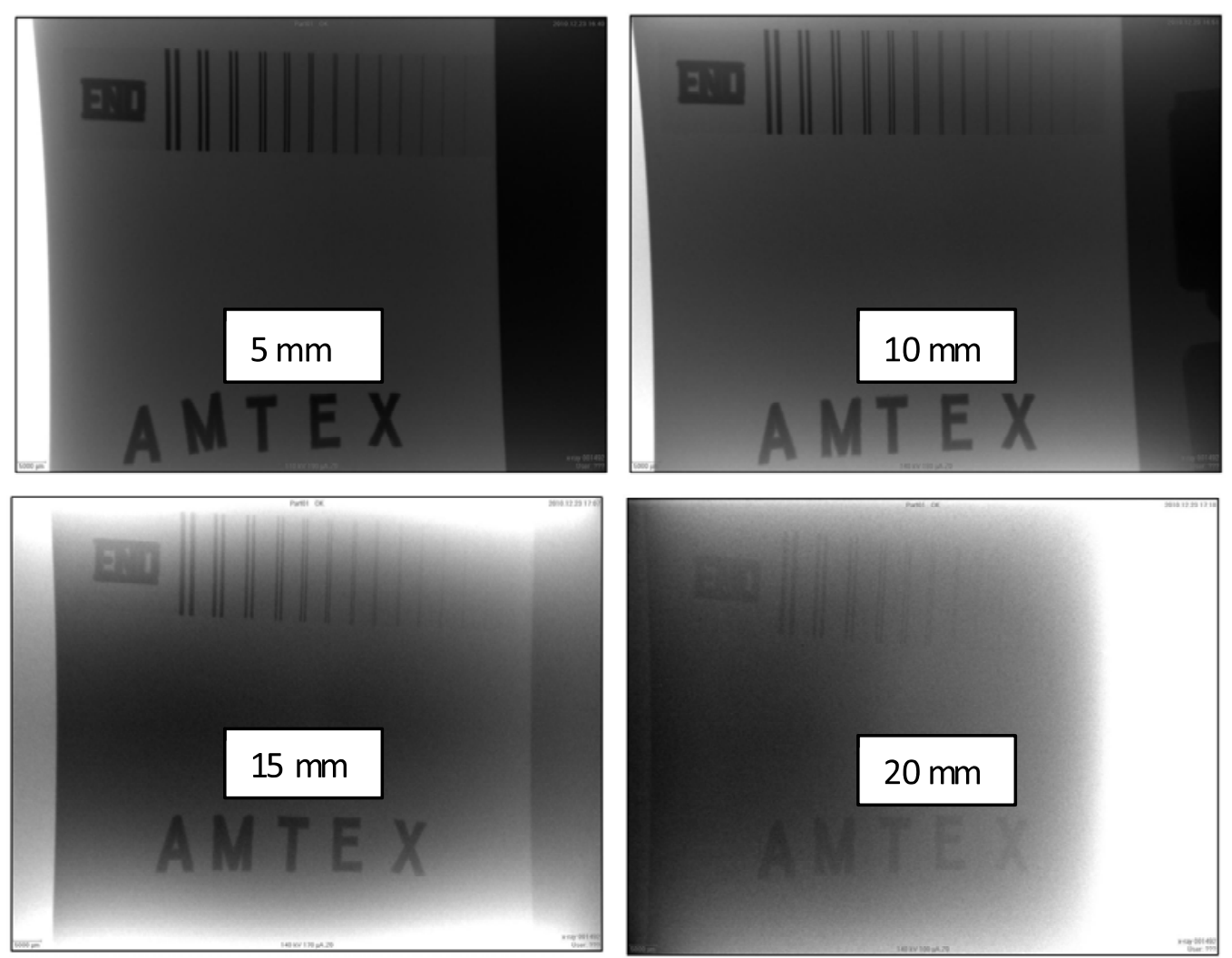

Figure 11. Step wedge on different thickness of material 

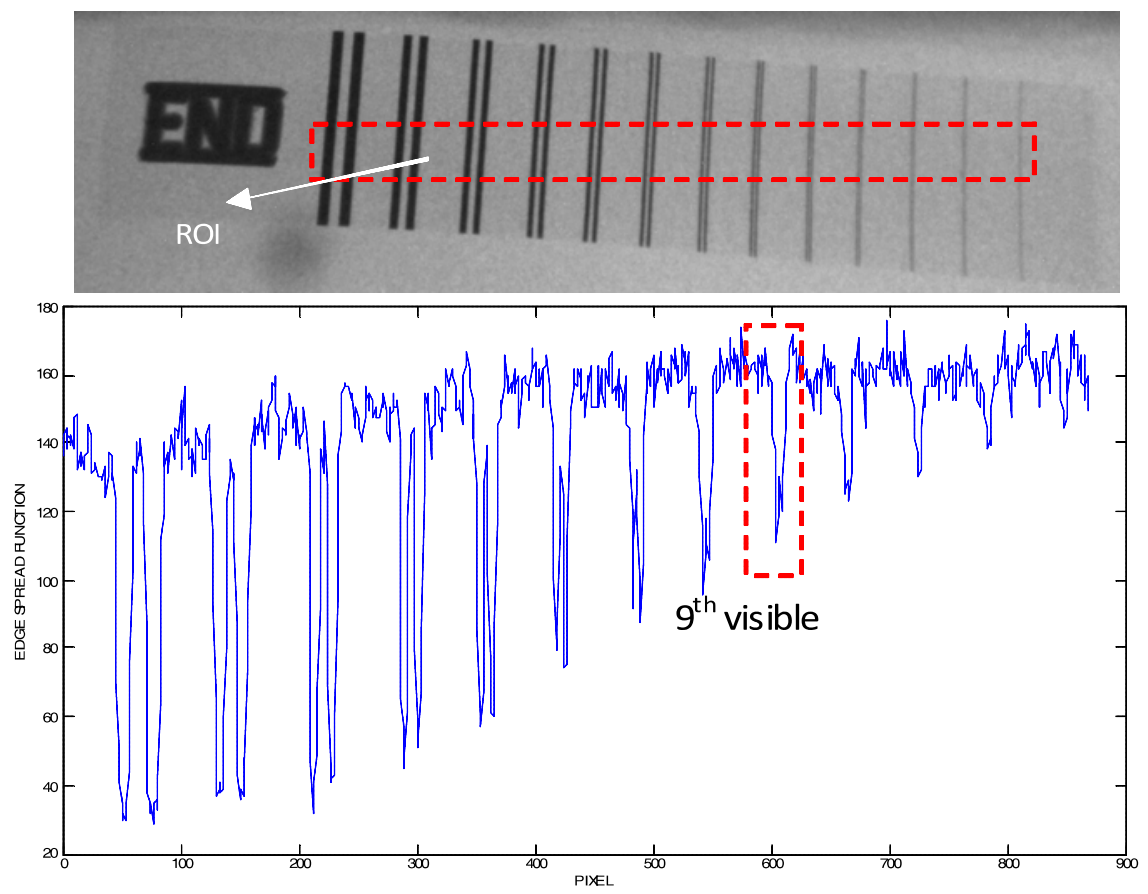

Figure 12. Region of interest (ROI) and edge spread function (ESF) curve for measuring the unsharpness of original image using IQI duplex wire

Table 1. Comparison between conventional and digital X-ray

\section{Conventional X-Ray}

Contrast is the difference in densities between various areas on a radiograph; high contrast images have few shades of gray between black and white while low contrast will demonstrate more shades of gray

Resolution is the ability to distinguish between small objects that are close together; measured in line pairs per millimeter

Density is the overall degree of darkening of an exp osed film

Sharpness is the ability of a radiograph to define an edge or display density boundaries

Require higher radiation exposure.

Focal Spot Size of conventional units is greater than 200 microns

Image acquired from the film directly without any special image processing techniques that can enhance overall image.

\section{Digital X-Ray}

Contrast resolution is the ability to differentiate small differences in density as displayed on an image

Spatial frequency is to measure of resolution expressed in line pairs per millimeter. MTF is to measure image fidelity as a function of spatial frequency (How close is the image to the actual object?).

Brightness is equivalent to density or overall degree of image darkening.

Signal to noise ratio-ratio (SNR) between the fraction of the output signal (voltage or current or charge) that is directly related to the information (signal) and the fraction of output that does not contain information (noise).

Less radiation exposure can be used to produce an image of similar con trast to conventional radiography.

Focal Spot Size of $\mu$-focused digital radiography is smaller than 50 microns which is enable to achieve the best resolution.

Immediate image preview and availability, a wider dynamic range which makes it more forgiving for overand under-exposure as well as the ability to apply special image processing techniques that enhance overall image. 
Table 2. IQI single wire table ( $10 \mathrm{FE} \mathrm{EN})$ for contrast sensitivity calculation and IQI duplex wire table (EN 462-5) for unsharpness and $\mathrm{SR}_{\mathrm{b}}$ calculation
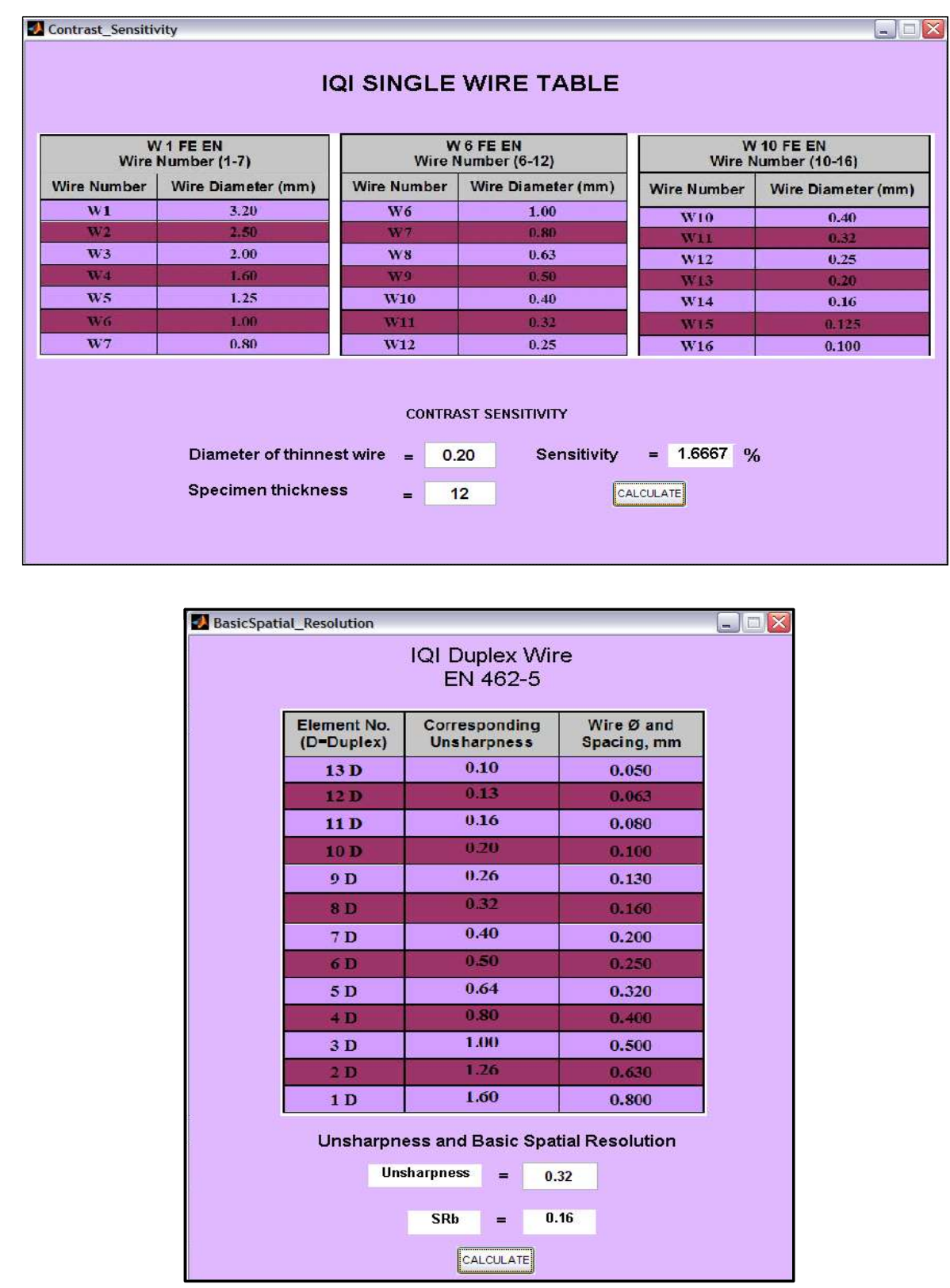

Table 3. Parameters used to capture the radiographed image of flawed specimen

\begin{tabular}{cccccc}
\hline \multirow{2}{*}{ Method } & \multicolumn{5}{c}{ Parameter } \\
\cline { 2 - 6 } & Voltage & Current & $\begin{array}{c}\text { Exposure } \\
\text { Time }\end{array}$ & FDD & FOD \\
\hline Unsharpness and MTF & $100 \mathrm{~V}$ & $160 \mathrm{~A}$ & 333 & $847 \mathrm{~mm}$ & $610 \mathrm{~mm}$ \\
Contrast Sensitivity & $150 \mathrm{~V}$ & $130 \mathrm{~A}$ & 100 & $590 \mathrm{~mm}$ & $350 \mathrm{~mm}$ \\
\hline
\end{tabular}


Table 4. Results of image quality for five noise removal methods

\begin{tabular}{ccccccc}
\hline \multirow{2}{*}{$\begin{array}{c}\text { Image Quality } \\
\text { Methods }\end{array}$} & Original & \multicolumn{5}{c}{ Noise Removal Method } \\
\cline { 3 - 7 } & & Median & Gaussian & Average & Disk & Motion \\
\cline { 3 - 7 } MSE & - & 16.8501 & 13.1209 & 19.1809 & 23.3542 & 24.7383 \\
PSNR & - & 35.8648 & 36.9512 & 35.3021 & 34.4471 & 34.1971 \\
MTF (lp/mm) & 15.92 & 16.31 & 17.38 & 16.34 & 17.67 & 19.75 \\
Unsharpness & 0.32 & 0.32 & 0.32 & 0.32 & 0.32 & 0.40 \\
SRb & 0.16 & 0.16 & 0.16 & 0.16 & 0.16 & 0.20 \\
Sensitivity (\%) & 1.6667 & 1.6667 & 2.6667 & 1.6667 & 1.6667 & 2.0833 \\
\hline
\end{tabular}

Table 5. Results of SNR and $\mathrm{SNR}_{\text {norm }}$

\begin{tabular}{ccccccc}
\hline & \multicolumn{5}{c}{ Results } \\
\cline { 2 - 7 } Image & $\begin{array}{c}\text { Thickness } \\
(\mathrm{mm})\end{array}$ & $\begin{array}{c}\text { Exposure } \\
\text { Time }\end{array}$ & Voltage & Current & SNR & SNR $_{\text {no rm }}$ \\
\cline { 2 - 7 } Image 1 & 5 & 67 & 110 & 190 & 20.4519 & 11.3252 \\
Image 2 & 10 & 100 & 140 & 180 & 19.0526 & 10.5504 \\
Image 3 & 15 & 200 & 140 & 170 & 5.564 & 2.4649 \\
Image 4 & 20 & 333 & 140 & 100 & 6.7594 & 1.4972 \\
\hline
\end{tabular}

\section{Acknowledgment}

The authors would like to express gratitude to the staff of the Faculty of Mechanical Engineering, Laboratory of Advanced Manufacturing and Advanced Manufacturing Technology Excellence Centre (AMTEx), Universiti Teknologi MARA (UiTM), Shah Alam for the technical support during this investigation. This investigation is financially sponsored by EScience MOSTI (Malaysian Ministry of Science and Technology), project number: 03-01-01 SF0355.

\section{References}

Edwin EP, Williamson GF (2002), Digital radiography: An Overview. The Journal of Contemporary Dental Practice 3(4):23-39.

Ewert U, Zscherpel U, Klaus B (2007), Strategies for film replacement in radiography- a comparative study. IV Conferencia Panamericana de END, Buenos Aires.

Han X, Deng F, Yin X (2009), An approach of adaptive enhancement of X-ray welding image.
International Conference on Information Engineering and Computer Science (ICIECS) 14.

Hendee WR, Russell RE (2002), Medical imaging physics. Fourth edition, ISBN: 0-471-38226-4, Copyright C_2002 Wiley-Liss, Inc.

Nadila PZ, Manurung YHP (2010), Advanced ndt using $\mu$-focussed digital radiography for welding inspection. $1^{\text {st }}$ Conference of National Postgraduate Seminar NAPAS, UiTM.

Noorhazleena (2010) Computed radiography (CR) signal to noise ratio (SNR) study based on thickness changes of steel step wedge. Journal of Malaysian Society for Non-Destructive Testing, MSNT.

Pardikar RJ (2008), Digital radiography and Computed radiography for Enhancing the Quality and Productivity of Weldments in Boiler components. 17th World Conference on Nondestructive Testing, Shanghai, China.

Rafael CG, Richard EW (1992), Digital image processing. Addison- Wesley Publishing Company.

Samei E, Flynn MJ (2003), An experimental comparison of detector performance for direct and indi- 
rect digital adiography systems. Medical Physics 30(4):608-622.

Samei E, Fynn MJ, Reimann DA (1998), Measuring the pre sampled MTF of Digital Radiographic Systems. J. of Medical Physics 25(1):102-113.

Uwe E, Uwe Z, Klaus B (2007), Possibilities and limits of digital industrial radiology - The new high contrast sensitivity technique - Examples and system theoretical analysis. Int. Symposium on Digital industrial Radiology and Computed
Tomography, Lyon, France.

Yeong-Taekgi M (1997), Contrast enhancement using brightness preserving bi-histogram equalization. IEEE Transactions on Consumer Electronics 43(1).

Xie XXX, Shi ZSZ, Guo WGW, Yao SYS (2009), An adaptive image enhancement technique based on image characteristic. 2nd Int. Congress on Image and Signal Processing 1-5. 